\title{
Evolución hacia la era digital de la cirugía ortognática. Experiencia en un centro universitario
}

\author{
Cristian Erazo C. ${ }^{1,2}$, Maritza Maripangui D. ${ }^{1}$, Diego Quispe V. ${ }^{1}$, Rolando Schulz R. ${ }^{1}$, \\ Rocío Jara ${ }^{1}$, Patricio Andrades C. ${ }^{1}$, Stefan Danilla E. ${ }^{1,2}$, Claudia Albornoz G. ${ }^{1}$, \\ Naomi Yamada T. ${ }^{1}$, Sofia Serra D. ${ }^{1}$ y Sergio Sepúlveda P. ${ }^{1,2}$
}

'Departamento de Cirugía Hospital Clínico Universidad de Chile. Santiago, Chile. ${ }^{2}$ Clínica Alemana de Santiago. Santiago, Chile.

Recibido 2019-08-29 y aceptado 2019-09-27

Correspondencia a Dr. Cristian Erazo C Cristian.erazo@gmail.com
Evolution towards the digital era of orthognatic surgery. Experience in a university center

Introduction: Technological advances have been incorporated into cranio-maxillo-facial surgery changing the clinical practice of surgeons. Aim: The aim of this article is to describe our experience in orthognatic surgery in the last 9-years, with the incorporation of 3D cephalometry. Materials and Method: A retrospective chart review was performed from January 2011 to August 2018 on patients undergoing Orthognatic Surgery. Demographic and surgical data, type of surgical planning, complications and long-term results were recorded. Results: A total of 21 patients underwent orthognatic surgery. Average age was 28 years (DE 8.1), 11 men (52.3\%) and 10 women (47.61\%). The principal surgeries performed were: $16(76 \%)$ bimaxillary, 3 maxillary advancement surgery $3(14 \%)$ and bilateral sagital split osteotomy $2(10 \%)$. Ethiologies were: 14 patients (this $67 \%$ ) with malocclusion class III, 6 patients $(28 \%)$ with malocussion class II, and 1 patient $(5 \%)$ with mandibular deviation. Virtual $3 \mathrm{D}$ presurgical planning was used in 11 patients $(52 \%)$. The total of complications Clavien-Dindo > III was $4.8 \%(n=1)$. None patient required reoperation. Satisfaction rate with the procedure was high. Conclusions: Based on our experience, orthognatic surgery techniques are safe. The complication rate was lower and well tolerated by patients. The introduction of virtual planning provided a better scenario to develop maxillo-facial surgery.

Key words: orthognatic surgeries; plastic surgery; postoperative complications.

\section{Resumen}

Introducción: La incorporación de tecnologías en la práctica quirúrgica, ha cambiado la forma de enfrentar el proceso quirúrgico. Objetivo: Describir la experiencia de los últimos 9 años, en cirugía ortognática, con la incorporación de la cefalometría 3D. Materiales y Método: Se realizó una revisión retrospectiva de pacientes operados de cirugía ortognática durante el período enero de 2011 a agosto de 2018. Se registraron datos demográficos, quirúrgicos, tipo de planificación quirúrgica, complicaciones y resultados a largo plazo. Resultados: 21 pacientes requirieron cirugías ortognáticas. Se realizaron 16 cirugías bimaxilares (76\%), 3 cirugías de avance maxilar superior (14\%) y 2 cirugías de osteotomía sagital de rama (10\%). Las principales etiologías fueron: $67 \%$ maloclusión clase III $(\mathrm{n}=14), 28 \%$ maloclusión clase II $(\mathrm{n}=6)$ y $5 \%$ desviación mandibular $(\mathrm{n}=1)$. La planificación prequirúrgica virtual fue utilizada en 11 pacientes (52\%). La tasa de complicaciones Clavien-Dindo > III fue 4,8\% (n=1). Conclusiones: En nuestra experiencia, las técnicas de cirugía ortognática son seguras. Las complicaciones de la serie fueron escasas y bien toleradas por los pacientes. Durante los últimos años y, de acuerdo a los avances tecnológicos, la incorporación de la planificación quirúrgica virtual 3D favoreció el desarrollo de la cirugía ortognática en nuestro centro.

Palabras clave: cirugías ortognáticas; cirugía plástica; complicaciones posoperatorias. 


\section{Introducción}

La íntima relación de la Cirugía Plástica y Cirugía Ortognática es de larga data. En el siglo IXX, el cirujano plástico Vilray Blair, en conjunto con el ortodoncista Edward Angle, reportaron la primera osteotomía de rama horizontal para el tratamiento del prognatismo ${ }^{1,2}$. Si bien en Chile la cirugía maxilofacial es una especialidad de la odontología, en otros países es una alternativa de especialización para médicos y odontólogos ${ }^{2,4}$. Los principales programas de cirugía plástica y reconstructiva consideran las competencias descritas por la Asociación Internacional de Cirujanos Orales y Máxilo-faciales para la formación de sus residentes en cirugía maxilofacial y su posterior ejercicio ${ }^{5-8}$. En algunos centros, la incorporación de ortodoncistas al equipo de cirugía plástica facilita el manejo de casos complejos y favorece la obtención de buenos resultados quirúrgicos. En nuestro país, el equipo de Cirugía Plástica y Reconstructiva del Hospital Clínico Universidad de Chile tiene una larga trayectoria en el campo de la cirugía maxilofacial. El Dr. Álvarez Salamanca desarrolló la cirugía plástica con especial énfasis en la cirugía ósea facial ${ }^{9}$. El año 1993, el Dr. Vicente de Carolis potenció la cirugía ortognática moderna y fue pionero en el uso de fotografía digital y cefalometría 2D computacional (QuickCef). Posteriormente, el Dr. Carlos Sciaraffia, aumentó el número de casos operados por el equipo. Actualmente, se realiza un trabajo interdisciplinario. El equipo se conforma por cirujanos plásticos formados en cirugía maxilofacial y un ortodoncista. Uno de los mayores avances que ha experimentado la cirugía ortognática en la última década ha sido la incorporación de la planificación quirúrgica virtual. El uso de la tomografía computarizada Cone-beam introducido al trabajo desde el año 2011, scanner ópticos intraorales y posterior diseño de férulas quirúrgicas mediante impresión $3 \mathrm{D}$, ha demostrado ser útil, confiable y reproducible en el diagnóstico, planificación y evaluación pre y posoperatoria cuando se compara con el método tradicional basado en cefalometría y modelos articulados de yeso ${ }^{10-12}$. La técnica quirúrgica también ha experimentado cambios durante la última década. Tradicionalmente, en cirugía bimaxilar se posiciona primero el maxilar superior y luego la mandíbula, sin embargo, existe evidencia que sugiere la inversión de esta secuencia $^{13,14}$

El objetivo de este estudio es describir la evolución de la cirugía ortognática en la experiencia del grupo en los últimos 9 años, con la incorporación de la cefalometría 3D.

\section{Materiales y Método}

\section{Recolección de datos}

Se realizó una revisión retrospectiva de fichas de los pacientes operados de cirugía ortognática durante el período enero de 2011 a agosto de 2018. Para cada paciente se registraron: edad, antecedentes médicos y quirúrgicos, diagnóstico, motivo de consulta, planificación prequirúrgica, tipo de cirugía, tiempo operatorio, complicaciones intra y postoperatorias. Se identificaron complicaciones en el intra y postoperatorio. Se consideraron mayores las clasificadas como Clavien Dindo mayor a III. La satisfacción con aspectos de la cirugía, fueron evaluados a través de una encuesta telefónica. El grado de alteración sensitiva del mentón fue evaluado a través de una escala graduada de 1 a $10(1=$ no siento nada, $10=$ siento completamente normal).

\section{Evaluación preoperatoria}

La evaluación integral preoperatoria de un paciente que requiere cirugía ortognática involucró 3 aspectos: médicos, odontológicos y psicológicos.

Una exhaustiva anamnesis y examen físico permitió la evaluación preoperatoria de patologías que pudiesen comprometer los resultados quirúrgicos (Ej. enfermedades sistémicas y síndromes genéticos).

La evaluación odontológica inicial se realizó por un ortodoncista (RS) con el fin de obtener una oclusión precisa luego de la cirugía ortognática. La orientación del crecimiento dentoalveolar, alineación de las piezas dentarías en sus bases maxilares y la creación de espacios para una rehabilitación protésica, fueron estrategias utilizadas para lograr la estabilidad de la oclusión postoperatoria.

Debido a los potenciales cambios en la fisonomía del paciente que conlleva la cirugía ortognática, se realizó una evaluación psicológica con el objetivo de clarificar expectativas del paciente e identificar patologías necesarias de tratar de forma concomitante o previa a la cirugía ortognática.

\section{Planificación quirúrgica}

La planificación prequirúrgica virtual fue utilizada en 11 pacientes (52\%). Estos fueron evaluados con un scanner maxilofacial y óptico intraoral (CEREC, Omniscann). El scanner maxilofacial tiene el objetivo de precisar la anatomía de los pacientes previo a la cirugía. El scanner óptico intraoral fue utilizado para mejorar la resolución de las arcadas dentarias.

Se realizó la planificación virtual preoperatoria utilizando el software Nemo Studio (v 11.0, 
Nemotec). El proceso involucró la reconstrucción tridimensional del esqueleto maxilofacial y arcos dentarios, lo que permitió preceder los cambios en el esqueleto óseo y oclusión. Posteriormente, se identificaron las medidas precisas para corregir las

Tabla 1. Características clínicas de los pacientes

\begin{tabular}{|c|c|}
\hline \multicolumn{2}{|l|}{ Edad, diagnostico y tipo de planificación quirúrgica } \\
\hline Total de pacientes & 21 pacientes \\
\hline - Hombres & 12 paciente $(57 \%)$ \\
\hline - Mujeres & 9 pacientes $(43 \%)$ \\
\hline Edad promedio & 28 años (DE 8,1) rango $18-49$ \\
\hline \multicolumn{2}{|l|}{ Diagnóstico: } \\
\hline - Malaoclusión Clase III & 14 pacientes $(67 \%)$ \\
\hline 9 Hombres (47\%): edad promedio 25 (DE 4,9) & Rango 18 - 33 \\
\hline 5 Mujeres (24\%): edad promedio 32 (DE 10,5) & Rango 19 - 48 \\
\hline - Malaoclusión Clase II & 6 Pacientes $(28 \%)$ \\
\hline 3 Hombres (14\%): edad promedio 32 (DE 15) & Rango 22 - 49 \\
\hline 3 Mujeres (14\%): edad promedio 29 (DE 2,7) & Rango $26-31$ \\
\hline - Asociado a mordida abierta esqueletal & 2 pacientes \\
\hline - Asociado a síndrome de la cara larga & 2 pacientes \\
\hline - Asociado a SAHOS & 1 paciente \\
\hline \multicolumn{2}{|l|}{$\begin{array}{l}*(1 \text { paciente presento malaoclusión II }+ \\
\text { Sd. cara larga }+ \text { mordida abierta esqueletal })\end{array}$} \\
\hline - Desviación mandibular izquierda & 1 paciente $(5 \%)$ \\
\hline \multicolumn{2}{|l|}{ Planificación prequirúrgica: } \\
\hline - Planificación virtual en 3D & 11 pacientes $(52 \%)$ \\
\hline - Planificación cefalométrica 2D & 10 pacientes $(48 \%)$ \\
\hline
\end{tabular}

Tabla 2. Distribución de cirugías ortognáticas

\begin{tabular}{|cc|}
\hline Tipo de cirugía & n (\%) \\
\hline - Cirugías bi-maxilares & $16(76 \%)$ \\
Secuencia maxila-mandíbula & $13(62 \%)$ \\
- Con genioplastía & $9(43 \%)$ \\
Secuencia mandíbula-maxila & $3(14 \%)$ \\
- Con genioplastía & $2(10 \%)$ \\
- Avance maxilar superior & $3(14 \%)$ \\
- Osteotomía sagital de rama mandibular & $2(10 \%)$ \\
- Rotación & $1(5 \%)$ \\
- Avance & $1(5 \%)$ \\
\hline
\end{tabular}

dismorfosis faciales, planificar los pasos de la intervención y diseñar la férula quirúrgica. La confección de las férulas quirúrgicas utilizadas en el intraoperatorio se confeccionaron utilizando la impresora 3D SLA resina (FormLabs 2).

En el pabellón, la férula se utilizó transitoriamente en cirugía combinada (ej. osteotomías bimaxilares) para asegurar una adecuada fijación durante la osteosíntesis. El acople oclusal definitivo se obtuvo a través de la ortodoncia preoperatoria.

\section{Técnica quirúrgica}

La técnica quirúrgica elegida fue individualizada para cada paciente, apuntando a la corrección estética y funcional en una sola intervención.

Se utilizaron técnicas clásicas de osteotomías: osteotomía tipo LeFort 1, osteotomía sagital de rama mandibular y osteotomía horizontal a nivel de la sínfisis del mentón (genioplastía). La osteotomía tipo LeFort I permitió intervenir el tercio medio facial. La osteotomía sagital de rama y genioplastía ósea fueron usadas para intervenir el tercio inferior. La elección y/o combinación de cada una de estas técnicas dependió del tipo de anomalía que presentó el paciente. La secuencia de la osteotomía en cirugías combinadas estuvo determinada principalmente por la comodidad del cirujano con la técnica quirúrgica empleada.

\section{Resultados}

Durante el periodo enero de 2011 a agosto de 2018 se operó un total de 21 pacientes. El promedio de edad fue de 28 años (DE 8,1), 11 varones y 10 mujeres. Las características demográficas de los pacientes se describen en la Tabla 1. El tiempo operatorio promedio de cirugía bimaxilar fue de $306 \mathrm{~min}$ (DE 63). El tiempo operatorio promedio en secuencia maxila-mandíbula fue de 305 min (DE 68) y 312 min (DE 42) en secuencia mandíbula-maxila. La distribución de las cirugías realizadas se evidencia en la Tabla 2 . No se identificaron complicaciones severas. Las complicaciones menores fueron: 1 fractura accidental de OSR 1 presentada en el intraoperatorio, 1 secuestro óseo y 1 gingivitis con retracción gingival. Un paciente presentó una complicación Clavien Dindo > III $(4,8 \%)$. El paciente presentó recurrencia de su dismorfosis debido a una caída con golpe de cara a los 14 días de operado. La encuesta telefónica fue respondida por 14 pacientes $(67 \%)$, operados hace 29,5 meses (rango 5,6-83,2). Los resultados de la encuesta telefónica se muestran en la Tabla 3. 


\section{Discusión}

Las deformidades dentofaciales (DDF) han sido descritas como alteraciones que principalmente afectan a los maxilares y los dientes, aunque puede ser afectada toda la cara. Tales deformidades pueden comprometer sólo la mandíbula o involucrar estructuras craneofaciales múltiples ${ }^{15}$. En la mayoría de los casos, son el resultado de distorsiones genéticas moderadas o severas del proceso de desarrollo normal y debe corregirse usando un protocolo integrado de ortodoncia durante el período de crecimiento y cirugía ortognática llegada la adultez ${ }^{14}$.

La prevalencia y el tipo de malaoclusión esqueletal varía entre distintos grupos raciales ${ }^{16}$. La oclusión clase III es más prevalente en poblaciones del Sudeste Asiático y menos prevalente en poblaciones angloamericanas. Se estima que un $15 \%-23 \%$ de los individuos de origen mongoloide presentan mala oclusión clase III $^{17-21}$. La población sudamericana tiene un fuerte componente mongoloide por lo que este mayor número de pacientes con oclusión clase III podría obedecer a la semejanza genética.

En un estudio realizado en Hong Kong ${ }^{22}$, en un período de 4 años, sobre un universo de 238 pacientes se encontró que el promedio de edad fue de 25,1 años, el $37,8 \%$ de los pacientes fueron de sexo masculino y el $62,2 \%$ femenino. El diagnóstico más frecuente en el maxilar fue la hipoplasia $(50 \%)$ y en la mandíbula hiperplasia $(32,8 \%)$, por lo que se puede inferir que el tipo de oclusión más prevalente fue de tipo III. Otro estudio realizado en población brasileña, examinando un período de 10 años, con 419 pacientes obtuvo datos similares al nuestro con una edad promedio en el momento de la cirugía de 28,5 años y un $55,9 \%$ de población femenina. El diagnóstico más frecuente fue la DDF tipo III esqueletal (41,3\%), le siguió DDF tipo II, clase I esqueletal, "cara larga" y "cara corta". Al igual que nuestro estudio, la cirugía más frecuentemente realizada fue la combinación de maxila, mandíbula y mentón en el $49,5 \%$ de los casos ${ }^{23}$.

En contraste a lo anterior, estudios epidemiológicos europeos revelan una mayor predominancia de la oclusión clase II. Panula y $\operatorname{cols}^{24}$, realizó una investigación en Finlandia, donde estudiaron un universo de 655 pacientes durante 13 años. Para ellos la media de edad fue de 30,3 años, con un $73 \%$ de mujeres y un $27 \%$ hombres. El $56 \%$ de los pacientes tenían una retrognatia mandibular. Otro estudio realizado en población neozelandesa durante un período de 8 años, sobre un universo de 92 pacientes, mostró que la mayoría de los pacientes fueron de sexo femenino en una relación de 1,6:1 y
Tabla 3. Alteraciones de la sensibilidad del mentón en pacientes operados de cirugía ortognática

$\begin{array}{ll}\text { - Alteración de la sensibilidad del mentón } & 14(100 \%) \\ \text { - Promedio de evaluación de sensibilidad } & \\ \text { en una escala de } 0 \text { a } 10^{*} & 6,9(\mathrm{DE} 2,3) \\ \text { - Mejoría del ronquido con la cirugía } & 3(21 \%) \\ \text { - La cirugía no modificó su ronquido } & 2(14 \%) \\ \text { - Nunca ronco } & 9(64 \%) \\ \text { - Grado de satisfacción } & 11(79 \% \text { de los encuestados) } \\ \text { - Muy satisfecho } & 2(14 \% \text { de los encuestados }) \\ \text { - Satisfecho } & 1(7 \% \text { de los encuestados) } \\ \text { - Insatisfecho } & \end{array}$

$* 0=$ No siento nada $; 10=$ Siento totalmente normal.

la cirugía más frecuente fue la de avance mandibular con un $54,3 \%{ }^{25}$.

En población chilena encontramos un único estudio que aporta información sobre epidemiología en cirugía ortognática. Pérez y $\operatorname{cols}^{26}$, en el año 2015, realizaron una revisión retrospectiva de los tratamientos quirúrgicos maxilofaciales en el Hospital San José, reportado cirugías ortognáticas en 71 pacientes, de los cuales $61 \%$ fueron mujeres y $39 \%$ hombres, sin embargo, no describen qué tipo de cirugía ortognática se realizó ni qué clase de oclusión tenían.

Nuestro estudio pone en evidencia la evolución de la planificación preoperatoria por medio de la planificación quirúrgica virtual $3 \mathrm{D}(\mathrm{PQV})$ y posterior confección de férulas quirúrgicas utilizando impresión 3D. Puesto que se realizó PQV en 52\% de los pacientes, se pudo efectuar una planificación más exacta de los movimientos de la maxila, (avance, impactación y canteo) así como los movimientos de avance, retroceso y ascenso de mandíbula en menor tiempo y esfuerzo por parte del equipo tratante (Figura 1). La PQV elimina muchos de los procesos de laboratorio dental que exige la planificación convencional con modelos, ya que, no es necesario realizar el trazado cefalométrico manual para predecir los movimientos óseos (2D) y no se requiere una réplica en yeso (modelos) de las arcadas dentarias para establecer la correcta relación del cráneo y del plano oclusal cuando se montan en el articulador. Esta exigente manipulación de la cirugía de modelos $(\mathrm{CM})$ puede inducir a error reduciendo la exactitud de la planificación ${ }^{10}$. En esta línea, Ritto y $\operatorname{cols}^{12}$, realizan una comparación de la exactitud diagnóstica de la posición de la maxila entre la planificación preoperatoria mediante $\mathrm{CM}$ y la PQV por medio de tomografía computada pre y posoperatoria superpuestas identificando que no 


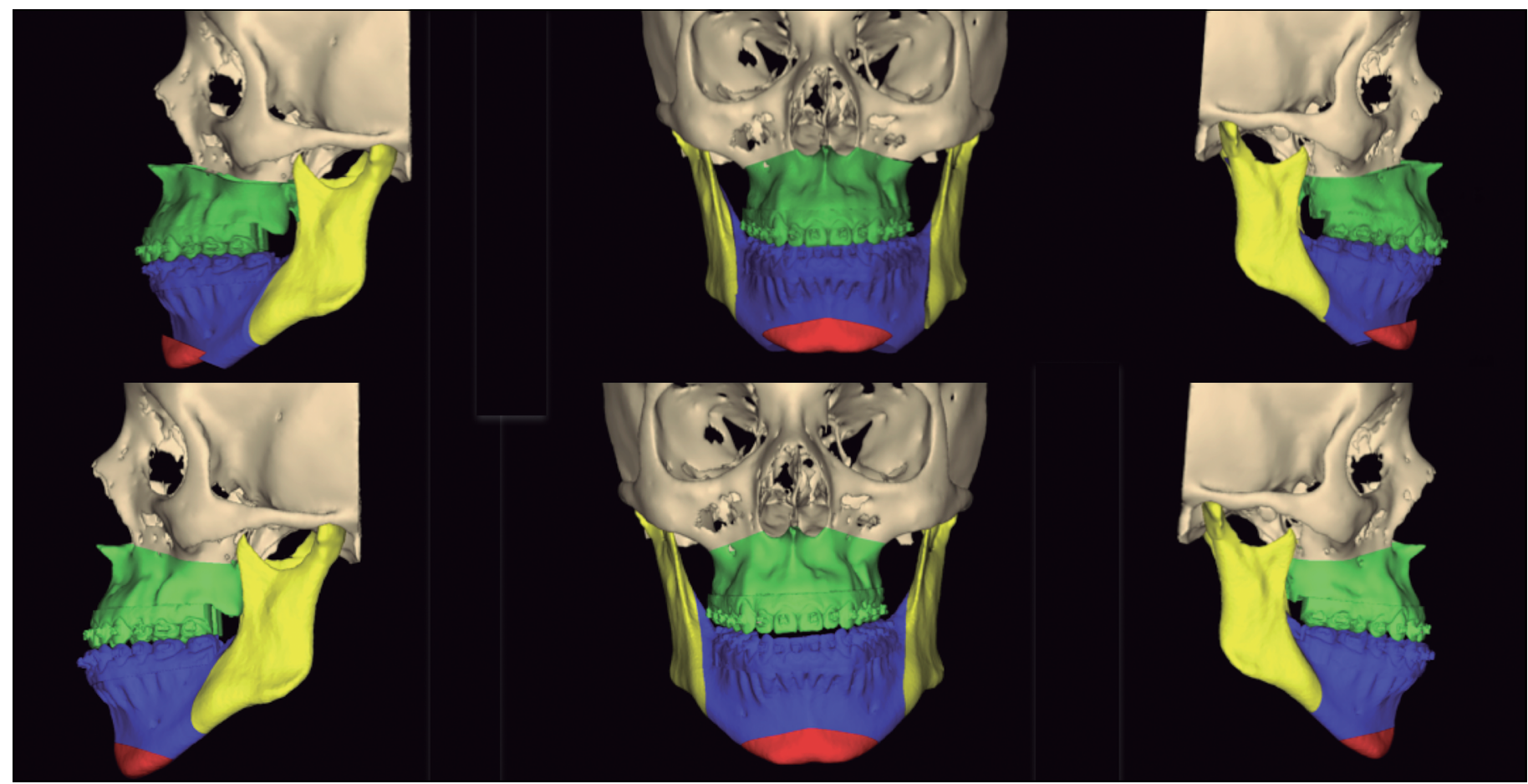

Figura 1. Planificación prequirúrgica paciente con diagnóstico de malaoclusión clase III 1. Panel superior: Planificación prequirúrgica virtual 3D. Panel inferior: reconstrucción virtual 3D de cirugía bimaxilar con medidas de avance para paciente.

había diferencia estadísticamente significativa en la exactitud diagnóstica de ambas. Resnick y cols ${ }^{27}$, efectuó una comparación del costo de realizar una PQV y posterior impresión 3D de las férulas versus evaluación cefalométrica en $2 \mathrm{D}$, modelo de cirugía y la fabricación manual de las férulas identificando que la PQV para cirugía bimaxilar utiliza significativamente menos tiempo y costos que la planificación convencional. Otro de los beneficios de la PQV es la capacidad de visualizar el el movimiento de los huesos y su influencia en los tejidos adyacentes, permitiendo discutir los resultados esperados con el paciente de manera que estos tengan expectativas más reales sobre éstos.

Con respecto a la técnica quirúrgica, una de las innovaciones del equipo en cirugías bimaxilares durante los últimos años es la secuencia de osteotomía mandíbula-maxila (Figuras 2 y 3). Esta

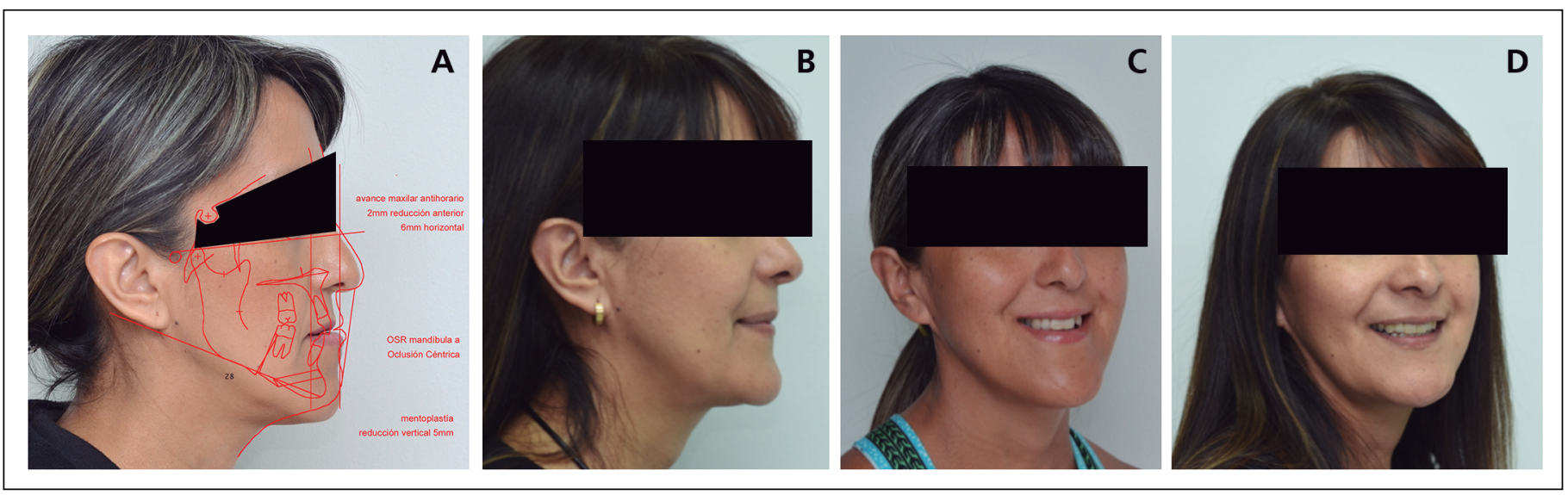

Figura 2. Fotografías pre y posoperatorias paciente Figura 1. A: Preoperatorio con trazados cefalométricos utilizados en planificación quirúrgica 2D. B: Postoperatorio cirugía bimaxilar en vista de perfil lateral. C: Preoperatorio en vista perfil 3/4. D: Postoperatorio a 15 meses de cirugía en perfil 3/4. 
secuencia fue realizada en 3 pacientes dentro de los cuales 2 de ellos se acompañaron de genioplastía de avance. El desarrollo y disponibilidad de fijaciones rígidas internas estables ha permitido reposicionar la mandíbula primero con tranquilidad ${ }^{28}$, sin embargo, aún existe controversia con respecto a las reales ventajas que entrega esta secuencia. Borba y cols ${ }^{13}$, realizaron una revisión sistemática de la secuencia mandíbula-maxila en cirugía bimaxilar identificando que la decisión parece estar basada en la experiencia y preferencia del cirujano y que, si bien existen ventajas teóricas que apoyan la secuencia mandíbula-maxila se requieren, estudios confiables, con resultados a corto y largo plazo para establecerlas como norma. Naran y cols ${ }^{14}$, en su revisión sobre cirugía ortognática recomienda empezar solo con la corticotomía sagital de las mandíbulas, luego proceder a reposicionar la maxila mediante LeFort 1 y luego retomar la mandíbula y completar la osteotomía. La razón de esta secuencia sería que cuando se realiza la osteotomía LeFort 1 primero, la apertura bucal posterior necesaria para visualizar adecuadamente la osteotomía mandibular puede ser muy amplia, pudiendo involuntariamente movilizar la reposición de maxila previa, en cambio, completar la osteotomía sagital previamente preparada no requeriría una apertura bucal tan amplia, por lo que existe menor riesgo de alterar la posición de la maxila. En opinión del autor, cuando se realiza una cirugía bimaxilar, la OSR es de mayor dificultad técnica debido a la limitación del campo visual, por lo que es conveniente realizarla en una primera instancia, para así disminuir la probabilidad de error por mal posición condilar.

La incidencia de complicaciones de nuestra serie fue baja o de menor complejidad. Jędrzejewski y $\operatorname{cols}^{29}$, en 2015, realiza una revisión sistemática evaluando las complicaciones asociadas a cirugía ortognática. El estudio incluyó 44 artículos e identificó las siguientes complicaciones: Alteración de sensibilidad (50\%), alteraciones de la articulación témporo-mandibular $(13,6 \%)$, hemorragia $(9 \%)$, infección $(6,2 \%)$, error de osteotomía $(4,5 \%)$, recurrencia de la dismorfosis $(4,6 \%)$ y necrosis ósea $(2,3 \%)$. De este análisis se puede advertir que las complicaciones encontradas en este estudio ya están descritas. En nuestra serie 1 paciente presentó una fractura parcial accidental sólo de la corteza externa durante OSR unilateral, permitiendo una adecuada superposición ósea para su fijación con tornillos. Se presentó secuestro óseo de un pequeño fragmento en 1 paciente $(4,8 \%)$, el cual se eliminó en forma espontánea y se manejó adecuadamente con tratamiento antibiótico.

Los pacientes encuestados de esta serie (67\%), refirieron algún grado de alteración sensitiva en la región mentoniana, sin embargo, el grado de compromiso promedio fue leve (promedio 6,9). La evaluación de la sensibilidad de la región inervada por el nervio alveolar inferior (NAI) posterior a cirugía mandibular, por lo general es difícil, dado que el reporte por paciente no es cuantificable y, hasta la fecha, no hay reportes sistemas de evaluación universales para comparar estudios. Agbaje y cols ${ }^{30}$, realizan una revisión sistemática de la incidencia de lesión de NAI posterior a OSR y de evaluación de alteraciones neurosensoriales entre 1990 y 2013 describiendo que existe una extrema variación en los reportes de alteraciones del NAI, siendo la evaluación subjetiva el método más usado para evaluar déficit neurosensorial. Otro estudio realizado en la Universidad de Hong Kong, entre 2012 y 2013
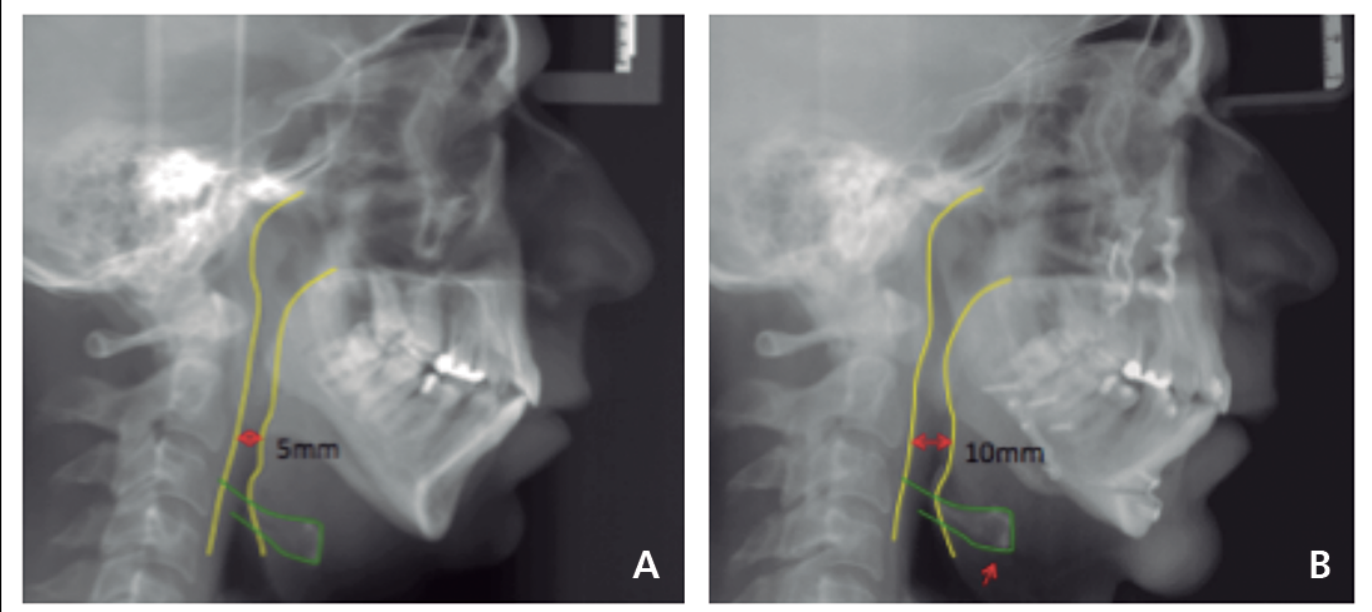

Figura 3. A: Radiografía de cuello lateral de paciente con diagnóstico de malaoclusión tipo II asociado a SAHOS en donde se aprecia una distancia anteroposterior de la hipofaringe de $5 \mathrm{~mm}$. B: Mismo paciente posterior a cirugía bimaxilar donde se aprecia una distancia anteroposterior de la hipofaringe de $10 \mathrm{~mm}$. 
evaluó la resolución del déficit neurosensorial en 66 pacientes de ambos sexos en los que se realizaron 132 procedimientos de rama mandibular. Los pacientes efectuaron un autorreporte del déficit sensorial durante los controles posoperatorios a las 2 y 6 semanas, 3 y 6 meses y 1 y 2 años. Este estudio concluye que el déficit neurosensorial del territorio de NAI después de cirugía ortognática se reduce progresivamente de un $78,8 \%$ a las 2 semanas a un $13,8 \%$ a $\operatorname{los} 2$ años posoperatorio ${ }^{31}$. En el caso de nuestro estudio, si bien solo se logró contactar a un $67 \%$ de los pacientes, el $100 \%$ de ellos presentaron alteraciones neurosensoriales de la región del mentón en grado leve. A pesar de las complicaciones presentadas, previamente descritas y acordes con la literatura, los pacientes estaban muy satisfechos o satisfechos con la cirugía.

\section{Conclusión}

En nuestra experiencia, las técnicas de cirugía ortognática son seguras. Las complicaciones de la serie fueron escasas y bien toleradas por los pacientes. Durante los últimos años y de acuerdo a los avances tecnológicos, la incorporación de la planificación quirúrgica virtual 3D favoreció la exactitud del plan quirúrgico y permitió la confección automatizada de férulas quirúrgicas, disminuyendo significativamente el tiempo y los costos comparado con la planificación convencional. La introducción de la planificación $3 \mathrm{D}$ favoreció la confianza para invertir el orden tradicional de la cirugía bimaxilar, iniciando el procedimiento con las osteotomías mandibulares.

\section{Responsabilidades éticas}

Protección de personas y animales. Los autores declaran que para esta investigación no se han realizado experimentos en seres humanos ni en animales.

Confidencialidad de los datos. Los autores declaran que en este artículo no aparecen datos de pacientes.

Conflictos de interés: no hay.

\section{Bibliografía}

1. Steinhäuser EW. Historical development of orthognathic surgery. J Craniomaxillofac Surg. agosto de 1996;24:195-204.

2. Stelnicki EJ, Young VL, Francel T, Randall P. Vilray P. Blair his surgical descendants, and their roles in plastic surgical development. Plast Reconstr Surg. 1999;103:1990-2009.

3. Maestre Rodríguez Ó, Monje Gil F, Cordero Acosta E. La cirugía oral y maxilofacial en la Unión Europea. Revista Española de Cirugía Oral y Maxilofacial 2013;35:150-6.

4. Cartes-Velásquez R, Ramírez $\mathrm{S} \mathrm{H}$, Cortés A J. Percepciones de los cirujanos maxilofaciales chilenos acerca del doble grado médico-dental. Revi Chil Cir. 2014;66:153-7.

5. International Association of Oral and Maxillofacial Surgeons. International Guidelines for Specialty Training and Education in Oral and Maxillofacial Surgery. En Durban; 2001. Disponible en: http://www.secom.org/wp-content/ uploads/2016/09/international guidelines specialty.pdf

6. Ortiz-Monasterio F, del Campo AF, Carrillo A. Advancement of the orbits and the midface in one piece, combined with frontal repositioning, for the correction of Crouzon's deformities. Plast Reconstr Surg. 1978;61:507-16.

7. Molina F, Ortiz Monasterio F. Mandibular elongation and remodeling by distraction: a farewell to major osteotomies. Plast Reconstr Surg. 1995;96:825-40; discussion 841-2.

8. Molina F. Mandibular Distraction Osteogenesis: A Clinical Experience of the Last 17 Years. Journal of Craniofacial Surgery 2009;20(Suppl 2):1794-800.

9. Andrades P. Cirugía plástica esencial. $1^{\text {a }}$ edición. Universidad de Chile; 2005.

10. Kwon TG, Choi JW, Kyung HM, Park HS. Accuracy of maxillary repositioning in two-jaw surgery with conventional articulator model surgery versus virtual model surgery. International Journal of Oral and Maxillofacial Surgery 2014;43:732-8.

11. Zhang N, Liu S, Hu Z, Hu J, Zhu S, Li Y. Accuracy of virtual surgical planning in two-jaw orthognathic surgery: comparison of planned and actual results. Oral Surgery, Oral Medicine, Oral Pathology and Oral Radiology 2016;122:143-51.

12. Ritto FG, Schmitt ARM, Pimentel T, Canellas JV, Medeiros PJ. Comparison of the accuracy of maxillary position between conventional model surgery and virtual surgical planning. International Journal of Oral and Maxillofacial Surgery 2018;47:160-6.

13. Borba AM, Borges AH, Cé PS, Venturi BA, Naclério-Homem MG, Miloro M. Mandible-first sequence in bimaxillary orthognathic surgery: a systematic review. International Journal of Oral and Maxillofacial Surgery 2016;45:472-5.

14. Naran S, Steinbacher DM, Taylor JA. Current Concepts in Orthognathic Surgery: Plastic and Reconstructive Surgery 2018;141:925e-936e.

15. Ong MA. Spectrum of dentofacial deformities: a retrospective survey. Ann Acad Med Singap. 2004;33:239-42.

16. Boeck EM, Lunardi N, Pinto A dos S, Pizzol KEDC, Boeck Neto RJ. Occurrence of skeletal malocclusions in Brazilian patients with dentofacial deformities. 
Brazilian Dental Journal 2011;22:340-5.

17. Tang ELK. The Prevalence of Malocclusion Amongst Hong Kong Male Dental Students. British Journal of Orthodontics 1994;21:57-63.

18. Yang WS. The study on the orthodontic patients who visited department of orthodontics, Seoul National University Hospital. Taehan Chikkwa Uisa Hyophoe Chi. 1990;28:811-21.

19. Singh GD, McNamara JA, Lozanoff S. Finite-element morphometry of soft tissues in prepubertal Korean and European-Americans with Class III malocclusions. Arch Oral Biol. 1999;44:429-36.

20. Watanabe M, Suda N, Ohyama $\mathrm{K}$. Mandibular prognathism in Japanese families ascertained through orthognathically treated patients. Am J Orthod Dentofacial Orthop. 2005;128:466-70.

21. van Vuuren C. A review of the literature on the prevalence of Class III malocclusion and the mandibular prognathic growth hypotheses. Aust Orthod J. 1991;12:23-8.

22. Alolayan AB, Leung YY. Risk factors of neurosensory disturbance following orthognathic surgery. PLoS ONE 2014;9:e91055.

23. Castro V, do Prado CJ, Neto AIT, Zanetta-Barbosa D. Assessment of the epidemiological profile of patients with dentofacial deformities who underwent orthognathic surgery. J Craniofac Surg. 2013;24:e271-275.

24. Panula K, Finne K, Oikarinen K. Incidence of complications and problems related to orthognathic surgery: a review of 655 patients. J Oral Maxillofac Surg. 2001;59:1128-36; discussion 1137

25. Parton AL, Tong DC, De Silva HL, Farella M, De Silva RK. A nine-year review of orthognathic surgery at the University of Otago. N Z Dent J. 2011;107:117-20.

26. Pérez Gutiérrez H, Donoso Hofer T, Mardones Muñoz M, Bravo Ahumada R. Epidemiología de Tratamientos Quirúrgicos Maxilofaciales en un Hospital Público en Santiago de Chile: Estudio Retrospectivo de 5 Años. International Journal of Odontostomatology 2015;9:3741.

27. Resnick CM, Inverso G, Wrzosek M, Padwa BL, Kaban LB, Peacock ZS. Is
There a Difference in Cost Between Standard and Virtual Surgical Planning for Orthognathic Surgery? Journal of Oral and Maxillofacial Surgery 2016;74:1827-33.

28. Pérez D, Ellis E. Implications of Sequencing in Simultaneous Maxillary and Mandibular Orthognathic Surgery. Atlas of the Oral and Maxillofacial Surgery Clinics 2016;24:45-53.

29. Jędrzejewski M, Smektała T, SporniakTutak K, Olszewski R. Preoperative, intraoperative, and postoperative complications in orthognathic surgery: a systematic review. Clinical Oral Investigations 2015;19:969-77.

30. Agbaje JO, Salem AS, Lambrichts I, Jacobs R, Politis C. Systematic review of the incidence of inferior alveolar nerve injury in bilateral sagittal split osteotomy and the assessment of neurosensory disturbances. International Journal of Oral and Maxillofacial Surgery 2015;44:44751.

31. Alolayan AB, Leung YY. Resolution of neurosensory deficit after mandibular orthognathic surgery: A prospective longitudinal study. Journal of CranioMaxillofacial Surgery 2017;45:755-61. 